\title{
Numerical Predictions of Cavitating Flow Around a Marine Propeller and Kaplan Turbine Runner with Calibrated Cavitation Models
}

\author{
Mitja Morgut $1{ }^{1}$ - Dragica Jošt ${ }^{2}$ - Aljaž Škerlavaj ${ }^{2}$ - Enrico Nobile ${ }^{1}$ - Giorgio Contento ${ }^{1}$ \\ 1 University of Trieste, Department of Engineering and Architecture, Italy \\ 2 Kolektor Turboinštitut, Slovenia
}

Cavitating phenomena, which may occur in many industrial systems, can be modelled using several approaches. In this study a homogeneous multiphase model, used in combination with three previously calibrated mass transfer models, is evaluated for the numerical prediction of cavitating flow around a marine propeller and a Kaplan turbine runner. The simulations are performed using a commercial computational fluid dynamics (CFD) solver and the turbulence effects are modelled using, alternatively, the Reynolds averaged Navier Stokes (RANS) and scale adaptive simulation (SAS) approaches. The numerical results are compared with available experimental data. In the case of the propeller the thrust coefficient and the sketches of cavitation patterns are considered. In the case of the turbine the efficiency and draft tube losses, along with the cavitation pattern sketches, are compared. From the overall results it seems that, for the considered systems, the three different mass transfer models can guarantee similar levels of accuracy for the performance prediction. For a very detailed investigation of the fluid field, slight differences in the predicted shapes of the cavitation patterns can be observed. In addition, in the case of the propeller, the SAS simulation seems to guarantee a more accurate resolution of the cavitating tip vortex flow, while for the turbine, SAS simulations can significantly improve the predictions of the draft tube turbulent flow.

Keywords: cavitation, marine propeller, Kaplan turbine, mass transfer models, RANS, SAS

Highlights

- CFD simulations of cavitating flow around a marine propeller and Kaplan turbine runner.

- Homogeneous model used in combination with three previously calibrated mass transfer models.

- Turbulence modelled using RANS and SAS approaches.

- Calibrated mass transfer models guarantee similar levels of accuracy.

- SAS approach improves the local flow field resolution.

\section{O INTRODUCTION}

In modern market scenarios, the competitiveness of an enterprise is determined, not only by the quality of the product but also by its time to market. As a matter of fact, nowadays, computational fluid dynamics (CFD) technologies are routinely used for design purposes allowing, in general, more expensive and time consuming experimental tests to be performed only at the final stages of the project. Such an approach becomes particularly relevant for parametric and/or optimization studies, where several simulations can be performed in parallel.

In the specific case of marine propellers and hydraulic turbines, CFD analysis can be effectively used to predict the overall machine performances as well as to investigate the effect of specific flow phenomena such as cavitation for instance [1] to [6].

Cavitation is the phenomenon that consists of the formation and activity of cavities (or bubbles) inside a liquid medium [7]. In flowing liquids it appears in low pressure regions where pressure, also owing to the system geometry, decreases below a certain threshold value. In the case of marine propellers and hydraulic turbines it is, usually, an undesirable phenomenon because in most cases it implies negative effects such as losses, efficiency reduction, noise, erosion and vibration [8] to [12].

In the last decades several CFD approaches have been developed to numerically investigate cavitating flow phenomena. A valuable review of different approaches is for instance provided by [13] and [14] and references therein. Among all the approaches, the most widely applied today is probably the socalled homogeneous transport-equation based model. In this approach the multiphase flow is treated as a homogeneous mixture of liquid and vapour, with variable density, and the relative motion between phases is neglected. The evaluation of the variable density field is based on an equation for void ratio with the source terms modelling the mass transfer rate due to cavitation, generally known as mass transfer model. In the literature there are available several mass transfer models relying on tunable parameters [14] even though interesting solutions for overcoming 
empiricism have for instance been proposed by [15] and [16].

In this study the homogenous transport- equation based model is considered and three different mass transfer models are employed. More precisely, the mass transfer models originally proposed by Zwart et al. [17], Singhal et al. [18] and Kunz et al. [19] with empirical coefficients calibrated according to [20] are employed.

The scope is to verify the applicability of the considered calibrated models to the numerical predictions of the cavitating flow around two different systems: marine propeller and Kaplan turbine.

The investigation is performed considering the Potsdam propeller test case (PPTC) model propeller working in uniform inflow [21], and a model Kaplan turbine experimentally investigated by researchers at Kolektor-Turboinstitut, Slovenia.

Even though the present study is carried out mainly to evaluate a possible more general character of the calibrated mass transfer models, related to the systems under consideration the influence of the turbulence modelling is also briefly evaluated. Thus, the simulations are performed using the standard Reynolds averaged Navier Stokes (RANS) approach and the more accurate and more time consuming Scale Adaptive Simulations (SAS). In the case of the steady state RANS simulations the workhorse Shear Stress Transport (SST) turbulence model [22] is used in combination with all the three different calibrated mass transfer models.

For the evaluation of the possible improvement related to a more accurate turbulence modelling approach, time dependent SAS simulations are carried out using the SST-SAS turbulence model [23] in combination with only a certain mass transfer model for convenience. The simulations are carried out using ANSYS-CFX (CFX for brevity) commercial CFD solver which is based on the node-centered finite volume method (more precisely on the Control Volume-Based Finite Element Method (CVFEM)) [24] and [25].

The numerical results are compared with the available experimental data. For a quantitative comparison the thrust is evaluated for the marine propeller, while the draft tube losses and the efficiency are considered for Kaplan turbine. For a qualitative comparison the sketches of cavitation patterns predicted around the blades are considered for both cases.

From this study it seems that for the prediction of the cavitating flow around a marine propeller and Kaplan turbine all the three different calibrated mass transfer models can be successfully employed. The machine performances can be predicted with a similar level of accuracy, even though small differences in the predicted cavitation patterns can be observed. As far as the turbulence modelling is concerned, the numerical results show that the SAS simulations could be used to improve the resolution of certain flow features such as the propeller cavitating tip vortex for example. Moreover, it seems that in the case of the Kaplan turbines, where the efficiency predictions are highly affected by the proper resolution of the unsteady draft tube turbulent structures, the SAS simulations could represent a good compromise between standard RANS simulations and the computationally more demanding and more accurate large eddy simulations (LES).

The paper is structured as follows. First the mathematical model is presented. Then, the numerical predictions performed for marine propeller and Kaplan turbine are described. The descriptions follow the same scheme where the considered system is presented, the numerical and meshing strategies are described, and the results are discussed. Finally, the concluding remarks are given.

\section{MATHEMATICAL MODEL}

Here, the homogeneous model is presented in the fixed frame of reference for convenience.

\subsection{Governing Equations}

In the homogeneous multiphase transport equationbased model, the cavitating flow can be described by the following set of governing equations:

$$
\left\{\begin{array}{l}
\nabla \cdot \mathbf{U}=\dot{m}\left(\frac{1}{\rho_{l}}-\frac{1}{\rho_{v}}\right) \\
\frac{\partial(\rho \mathbf{U})}{\partial t}+\nabla \cdot(\rho \mathbf{U U})=-\nabla P-\nabla \cdot \tau+S_{M} . \\
\frac{\partial \gamma}{\partial t}+\nabla \cdot(\gamma \mathbf{U})=\frac{\dot{m}}{\rho_{l}}
\end{array}\right.
$$

Cavitating flow is modelled as a mixture of two species i.e. vapour and liquid behaving as one. The phases are considered incompressible. They share the same velocity $\mathbf{U}$ and pressure fields $P$.

The mixture density, $\rho$, and dynamic viscosity, $\mu$, are scaled, respectively, as:

$$
\rho=\gamma \rho_{l}+(1-\gamma) \rho_{v}, \quad \mu=\gamma \mu_{l}+(1-\gamma) \mu_{v} .
$$


The interface mass transfer rate due to cavitation, $\dot{m}$, can be modelled using three different calibrated mass transfer models.

\subsection{Turbulence Modelling}

In order to model turbulence effects different approaches can mainly be applied, depending on the required accuracy and the available computational resources. In this study we adopted the standard RANS approach in combination with the workhorse SST turbulence model, and the more advanced SST-SAS model available in CFX. For a detailed description of the considered models we refer to [22], [23] and [26]. Here, we clarify that the SST-SAS model is an improved unsteady-RANS formulation, with the ability to adapt the length scale to resolved turbulent structures by including the von Karman length-scale into the turbulence scale equation. The information given by the von Karman length scale allows SST-SAS model to dynamically adjust to resolved structures mimicking a LES-like behaviour in unsteady regions of the flow field. At the same time, the model provides standard RANS capabilities in stable flow regions.

\subsection{Mass Transfer Models}

The mass transfer models employed in this study were previously calibrated using an optimization strategy, where selected empirical coefficients of the considered models, were properly tuned for the prediction of the sheet cavity flow around a hydrofoil [20]. In the following the formulations of the considered mass transfer models are provided, and in Table 1 the calibrated empirical values namely $F_{e}, F_{c}$, $C_{e}, C_{c}, C_{\text {prod }}, C_{d e s t}$, are collected.

Zwart et al. model:

$$
\dot{m}=\left\{\begin{array}{ll}
-F_{e} \frac{3 r_{n u c}(1-\alpha) \rho_{v}}{R_{B}} \sqrt{\frac{2}{3} \frac{P_{v}-P}{\rho_{l}}} & \text { if } P<P_{v} \\
F_{c} \frac{3 \alpha \rho_{v}}{R_{B}} \sqrt{\frac{2}{3} \frac{P-P_{v}}{\rho_{l}}} & \text { if } P>P_{v}
\end{array} .\right.
$$

Full cavitation model (FCM):

$$
\dot{m}=\left\{\begin{array}{ll}
-C_{e} \frac{\sqrt{k}}{\kappa} \rho_{l} \rho_{v} \sqrt{\frac{2}{3} \frac{P_{v}-P}{\rho_{l}}}\left(1-f_{v}\right) & \text { if } P<P_{v} \\
C_{c} \frac{\sqrt{k}}{\kappa} \rho_{l} \rho_{l} \sqrt{\frac{2}{3} \frac{P-P_{v}}{\rho_{l}}} f_{v} & \text { if } P>P_{v}
\end{array} .\right.
$$

Kunz et al. model:

$$
\dot{m}=\dot{m}^{+}+\dot{m}^{-}:\left\{\begin{array}{l}
\dot{m}^{+}=\frac{C_{\text {prod }} \rho_{v} \gamma^{2}(1-\gamma)}{t_{\infty}} \\
\dot{m}^{-}=\frac{C_{d e s t} \rho_{v} \gamma \min \left[0, P-P_{v}\right]}{\left(0.5 \rho_{l} U_{\infty}^{2}\right) t_{\infty}}
\end{array} .\right.
$$

Table 1. Calibrated model coefficients

\begin{tabular}{ccc}
\hline Model & Evaporation & Condensation \\
\hline Zwart & $F_{e}=300$ & $F_{c}=0.03$ \\
\hline FCM & $C_{e}=0.40$ & $C_{c}=2.3 \times 10^{-4}$ \\
\hline Kunz & $C_{\text {dest }}=4100$ & $C_{\text {prod }}=455$ \\
\hline
\end{tabular}

It is worth clarifying that the Zwart et. al model is the native CFX mass transfer model while the FCM and Kunz et al. models were additionally implemented using the cell expression language (CEL) available in CFX. In the case of the FCM, following [27], $f_{v}$ was replaced by $\alpha$. In all simulations the mass transfer rate was considered positive if directed from vapour to liquid phase and the maximum density ratio $\rho_{l} / \rho_{v}$ was clipped to 1000 for solver stability reasons.

\section{MARINE MODEL SCALE PROPELLER}

The numerical predictions for cavitating PPTC propeller working in uniform inflow are presented.

The considered propeller is a five-bladed, controllable pitch propeller having a diameter $D=0.250 \mathrm{~m}$. It was used as a blind test case at the 2011 Workshop on Cavitation and Propeller Performance. A significant amount of experimental data is currently available at [21].

\subsection{Numerical Strategy}

Due to the periodicity of the problem (uniform inflow and in this case neglected gravity) only one blade passage was modelled for computational convenience in all propeller simulations. Fig. 1 shows the shape of the computational domain. In Table 2 the values of the corresponding main dimensions are collected.

Since the propeller rotation was simulated using multiple reference frame (MRF) approach the computational domain was subdivided into two regions namely rotating and fixed. In Fixed the governing equations were solved by considering a fixed frame of reference, while in rotating, the governing equations were solved using a rotating frame of reference. 


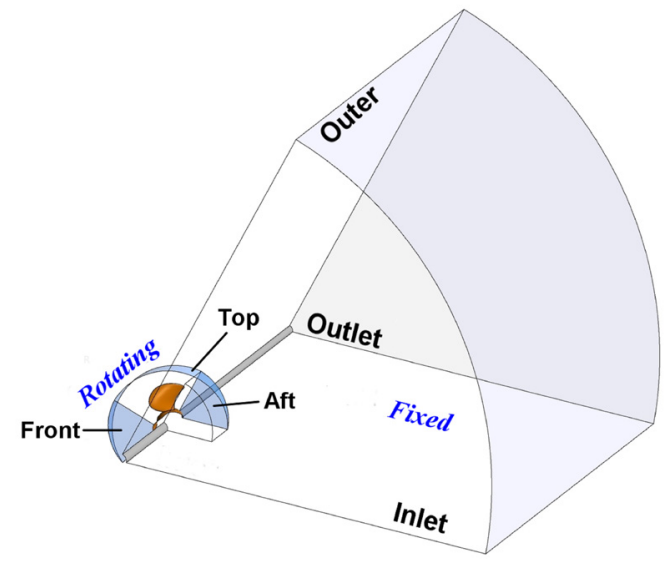

Fig. 1. Shape of the computational domain; rotating region surrounded by front, aft and top interfaces

The following boundary conditions were applied: on inlet boundary, the free-stream velocity components and a turbulence level of $1 \%$ were set. The freestream values (as well as the propeller rotational velocity) were set following the experimental setup [21]. The Reynolds number, $R e_{P}$, was in range (1.7 to 1.8$) \times 10^{6}$. On outlet boundary, a fixed value of the static pressure equal to $202,650 \mathrm{~Pa}$ was imposed. On the periodic boundaries (sides of the domain), the rotational periodicity was ensured. On solid surfaces the no-slip boundary condition was applied, and on outer boundary, the slip condition was set. Steady state RANS and unsteady SAS simulations were performed. In the case of the RANS simulations the workhorse SST turbulence model was used, while for SAS simulations the SST-SAS model was employed. Both models were used in combination with the automatic wall treatment available in CFX.

Table 2. Distances of the boundaries/surfaces from the propeller mid plane in axial direction for inlet, outlet, aft, and from the propeller rotation axis in radial direction for outer and top

\begin{tabular}{cccccc}
\hline Inlet & Outlet & Outer & Front & Aft & Top \\
\hline $2.30 \mathrm{D}$ & $5.30 \mathrm{D}$ & $5.00 \mathrm{D}$ & $0.41 \mathrm{D}$ & $0.31 \mathrm{D}$ & $0.60 \mathrm{D}$ \\
\hline
\end{tabular}

As far as the discretization of the advective terms is concerned, for the RANS simulations, the high resolution scheme was employed while a bounded second order central difference scheme was used in the SAS simulations. For time discretization a first order implicit time scheme was used. It is worth clarifying that in this study with SAS an almost stable cavitating-tip vortex flow was investigated (see Fig. 4).
Thus, we assumed that, for this specific case, the more stable first order time scheme can be conveniently used and ensure a similar level of accuracy as the generally more unstable second order scheme.

Table 3. PPTC propeller; thrust coefficient for RANS simulations with different mass transfer models

\begin{tabular}{cccccc}
\hline \multirow{2}{*}{$J$} & \multirow{2}{*}{$\sigma_{n}$} & \multirow{2}{*}{$K_{T, E X P}$} & \multicolumn{3}{c}{$K_{T, C F D}$} \\
\cline { 4 - 6 } & & & Zwart & FCM & Kunz \\
\hline 1.016 & 2.024 & 0.373 & 0.373 & 0.374 & 0.375 \\
\hline 1.269 & 1.424 & 0.206 & 0.196 & 0.203 & 0.210 \\
\hline 1.408 & 2.000 & 0.136 & 0.133 & 0.130 & 0.133 \\
\hline
\end{tabular}

\subsection{Meshing}

The computational grids for fixed and rotating were generated independently and then joined in CFX through the General Grid Interfaces (GGI) solver capabilities. Both meshes were hexa-structured and were created using ANSYS ICEM CFD (ICEM for brevity).

The overall mesh had about $2.1 \times 10^{6}$ nodes with a proper refinement in the tip vortex region following [29].

The considered mesh arrangement proved to guarantee mesh independent results in former studies [30]. The average $y^{+}$value on the blade surface was about 32. Fig. 2 shows the blade surface mesh.

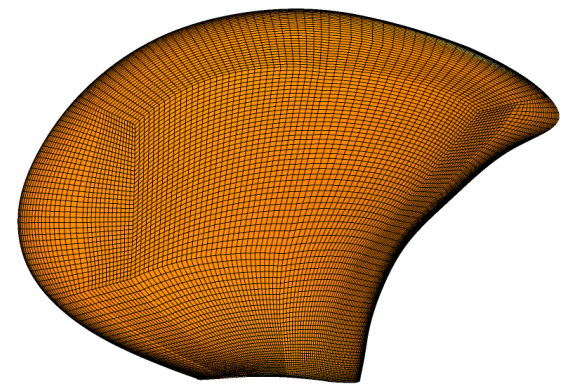

Fig. 2. PPTC propeller, blade surface mesh

\subsection{Results}

The simulations were carried out following the experimental setup suggested in [21]. The overall numerical predictions performed using the steadystate RANS approach compared well with the available experimental data.

Regarding the thrust (thrust coefficient) only minor differences were observed among the results obtained varying the mass transfer model. From Table 

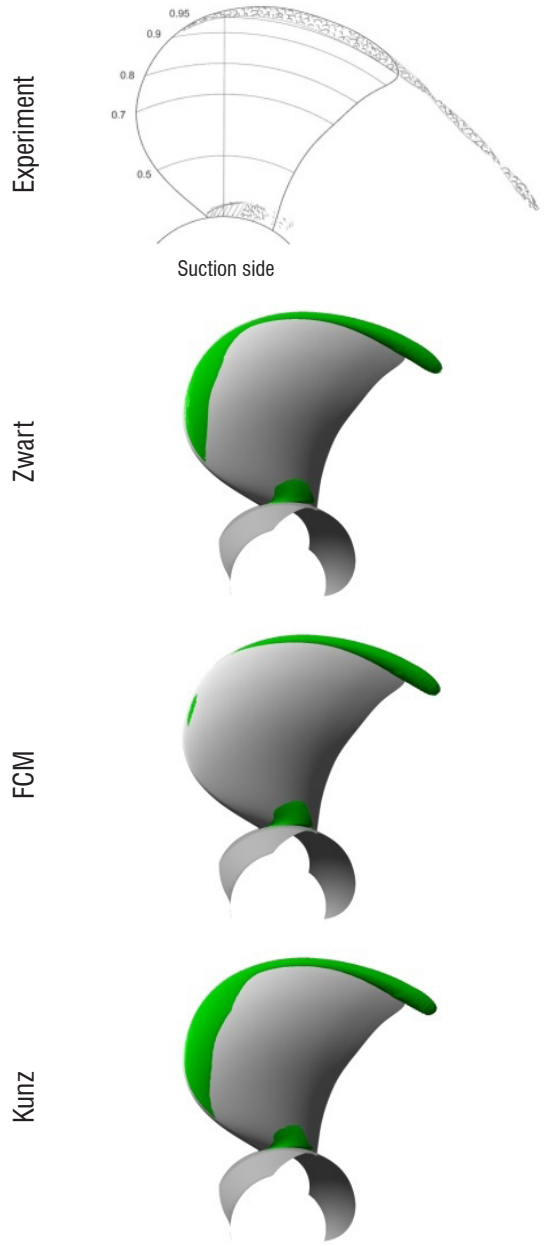

$J=1.019, \sigma_{n}=2.024$
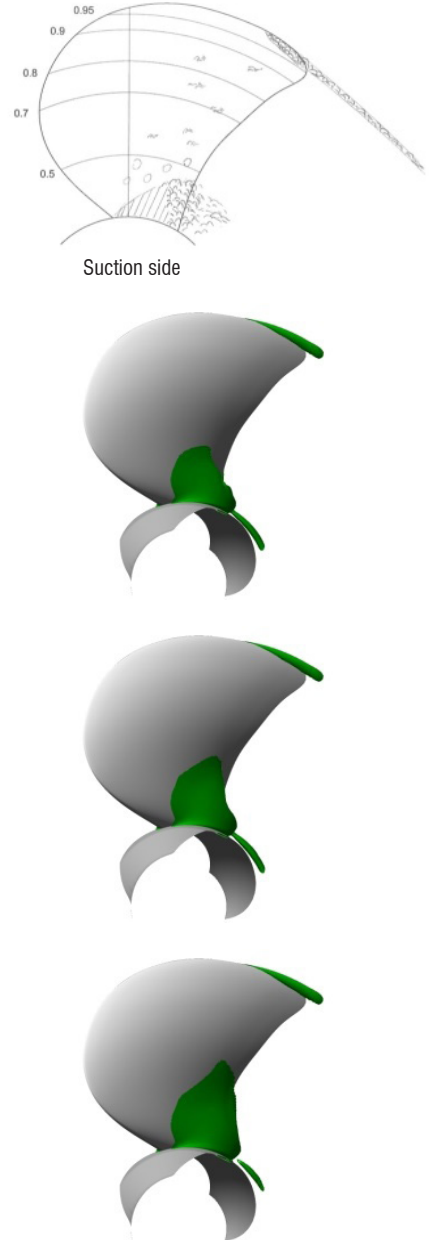

$$
J=1.269, \sigma_{n}=1.424
$$
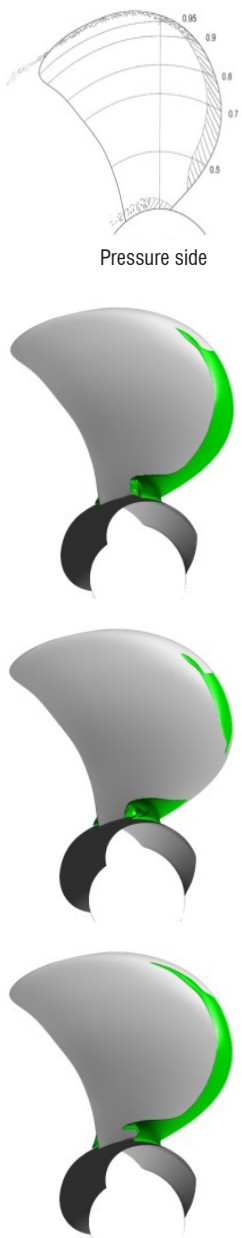

$J=1.408, \sigma_{n}=2.000$

Fig. 3. PPTC propeller; RANS simulation performed with three different mass transfer models; cavitation patterns depicted using isosurfaces of vapour volume fraction equal to 0.2

3 it is possible to note that for a given operational condition the thrust predicted using the three different calibrated mass transfer models was in excellent agreement with the experimental data.

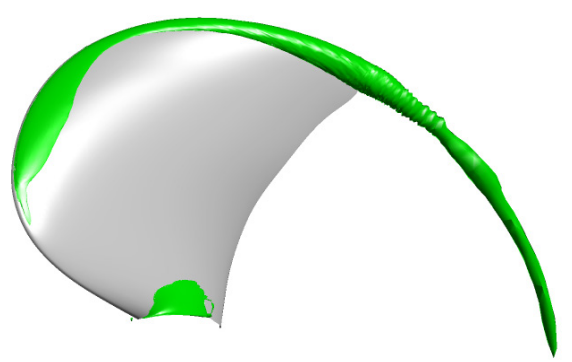

Fig. 4. Cavitation pattern predicted using the SST-SAS turbulence model in combination with the calibrated FCM mass transfer model for $J=1.019, \sigma_{n}=2.024$
Following [21] the cavitation patterns are here presented as isosurfaces of vapour volume fraction equal to 0.2 . From the qualitative comparison of the snapshots of the cavitation patterns, presented in Fig. 3 it is interesting to observe that for $J=1.019$, $\sigma_{n}=2.024$, only in the case of the FCM the shape of the cavitation pattern was correctly reproduced. With the other models a layer of sheet cavitation on the blade leading edge, not observed experimentally, was obtained. Conversely, for $J=1.408, \sigma_{n}=2.000$, the extent of the sheet cavity developing on the propeller face was better reproduced with the Zwart and Kunz models. The extent of the cavitation pattern predicted with the FCM was minor. The reasons behind these differences are still not fully clear. For $J=1.269$, $\sigma_{n}=1.424$ there were no differences in the cavitation 
patterns predicted using the three different mass transfer models.

Since in the case of the RANS simulations the tip-vortex was slightly under-estimated, in this study, focusing on $J=1.019, \sigma_{n}=2.024$, a brief evaluation of the SAS simulation was performed. An additional SAS simulation was performed in combination with the FCM model, even though the other two models could also be adopted for this purpose. The FCM was used, mainly, because in the former RANS simulation the most accurate prediction of the cavitation pattern, for the specific operational condition, was obtained using this mass transfer model. Fig. 4 shows that with the SAS simulation the extension of the cavitating tip-vortex was better reproduced. This improvement is related to the less diffusive character of the SAS simulations where, in general, lower levels of the turbulent viscosity are predicted than those obtained with the corresponding RANS simulations.

\section{MODEL SCALE KAPLAN TURBINE}

The numerical predictions of the cavitating flow in a model scale medium head Kaplan turbine are presented.

The turbine in question was developed by Kolektor-Turboinstitut and consists of a semi-spiral casing with two vertical piers, 11 stay vanes and a nose, 28 guide vanes, a 6-blade runner, and an elbow draft tube with two vertical piers. In CFD simulations a draft tube prolongation was added to improve solver stability as depicted in Fig. 5.

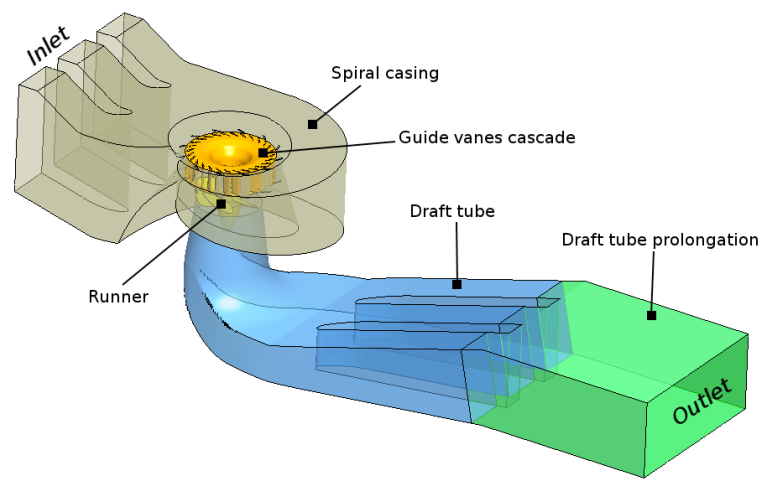

Fig. 5. Sketch of the Kaplan turbine used in simulations

The experimental tests were performed on the test rig at Kolektor-Turboinstitut following the IEC 60193 [31] international standard.

In this study the effect of the severity of the cavitating phenomena was analysed considering an operating point, close to the local best efficiency point, determined by a certain combination of guide vane blade opening angle and rotor blade angle. For such a point the flow and energy coefficients were $\varphi / \varphi_{B E P}=1.33$ and $\psi / \psi_{B E P}=0.86$, respectively. The Reynolds number, $R e_{T}$, was $6 \times 10^{6}$.

\subsection{Numerical Strategy}

All the simulations were carried out considering the computational domain shown in Fig. 5.

Similarly to the propeller case, the numerical investigations were first performed using the steady state RANS approach, mainly to evaluate the effect of the calibrated mass transfer models on the accuracy of the numerical predictions. Then, further SAS simulations were carried out in order to improve efficiency predictions.

For RANS simulations the SST turbulence model was used while for SAS simulations the SST-SAS model was employed. Both were used in combination with the automatic wall treatment. Moreover, the curvature correction [32] and the Kato launder production limiter [33] were, here, included in all the simulations. For the discretization of the advective terms high resolution method was used for both RANS and SAS simulations. The use of the second order bounded central difference scheme (BCDS) in the current SAS simulations was precluded by poor solver stability. For time discretization a second order implicit time scheme was used.

For cavitation modelling all the three different mass transfer models were employed in RANS simulations while for SAS simulations the calibrated Zwart model was used exclusively.

It is important clarifying that during the design process of the current turbine, carried out by KolektorTurboinstitut, the standard Zwart model available in CFX was used. Therefore the SAS simulations were carried out in combination with the calibrated Zwart model in order to verify the benefits, in terms of accuracy, of using an advanced URANS model like SAS with the same (calibrated) mass transfer model [35].

The computational domain was properly subdivided in rotating region containing the runner and in fixed region including the rest of the turbine parts. In the case of the steady state RANS simulations the MRF approach was employed and at the interfaces between rotating and fixed regions the frozen rotor frame change/mixing model was used. In the case of SAS simulations the sliding grid approach (transient rotor stator) available in CFX was used. 
All the simulations were carried out by imposing a given flow rate corresponding to a given flow rate coefficient. Thus, on inlet boundary a proper velocity distribution with a $5 \%$ turbulence level was set. On solid surfaces the no-slip wall boundary condition was imposed.

Although, the effect of gravity for the model size is small, in this study, gravity was included in computations for the sake of completeness. Therefore, a value of static pressure prescribed at outlet boundary also included the hydrostatic pressure.

In current simulations the tip clearance was modelled while the hub clearance was neglected.

\subsection{Meshing}

The computational grid was composed of several turbine parts. It had about $8.3 \times 10^{6}$ nodes distributed as shown in Table 4.

Table 4. Turbine mesh characteristics

\begin{tabular}{lc}
\hline Turbine part & Nodes \\
\hline Semi spiral casing with stay vanes & $1,480,999$ \\
\hline Guide vane cascade & $2,755,496$ \\
\hline Runner & $1,858,374$ \\
\hline Draft tube & $1,786,432$ \\
\hline Draft tube prolongation & 398,056 \\
\hline Total & $8,279,357$ \\
\hline
\end{tabular}

The meshes, for the different turbine parts, were generated independently of each other and subsequently joined in CFX using GGI. The meshes were created using ICEM and Turbogrid.

The grid in the spiral casing with stay vanes was unstructured, while the grids in the other turbine parts were structured. The average $y^{+}$value on the different turbine parts was in the range of 3 to 9 . The suitability of the considered mesh was verified in former studies [34]. Fig. 6 shows snapshots of surface meshes for the runner, draft tube and draft tube prolongation.

\subsection{Results}

In Fig. 7 the curves of efficiency and draft tube losses (head) are presented.

Considering the efficiency, it is interesting to note that similarly to the propeller case the simulations performed using different calibrated mass transfer models guaranteed similar results. As a matter of fact, from a qualitative comparison presented in Fig. 8 it is possible to note that the cavitation patterns obtained with different calibrated models were very similar to

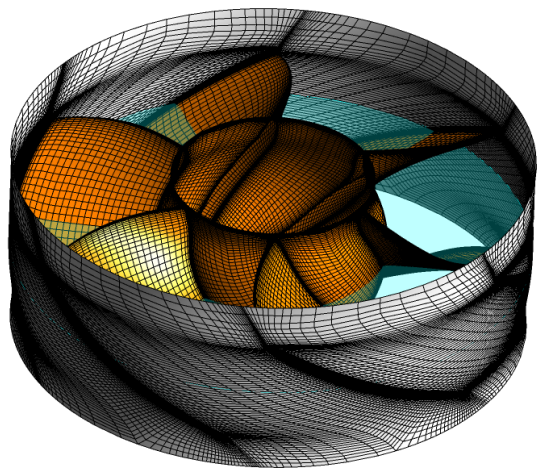

a)

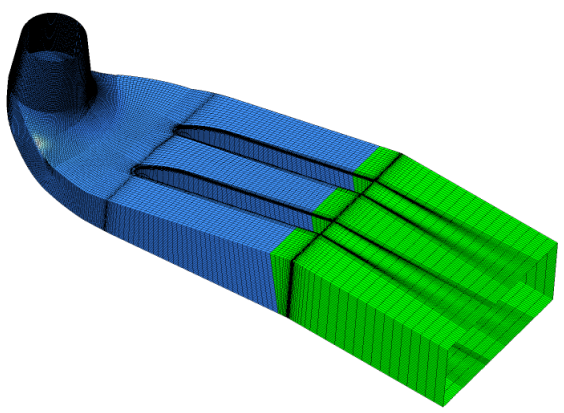

Fig. 6. Surface meshes; a) runner, and b) draft tube and draft tube prolongation

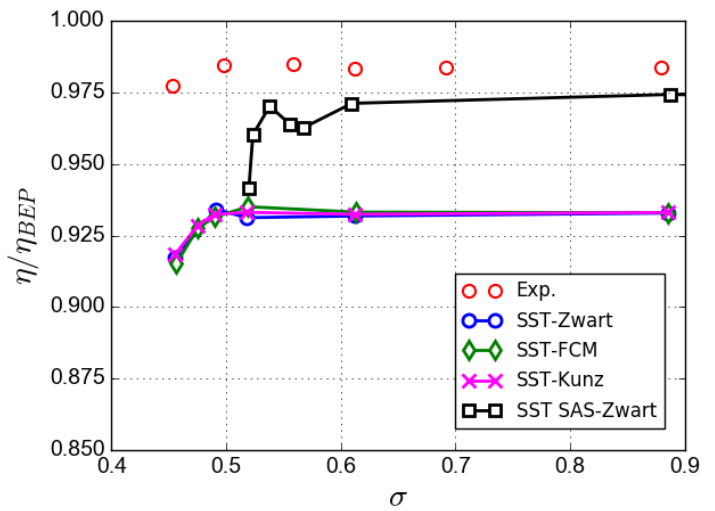

a)

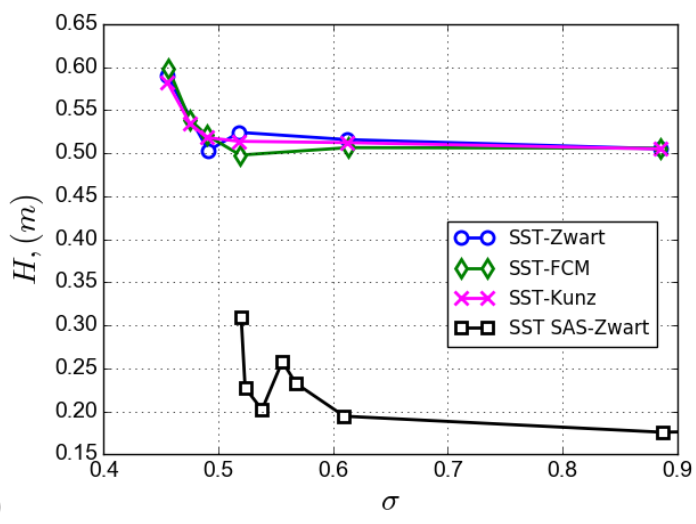

Fig. 7. a) Efficiency and b) draft tube losses, predicted using steady state simulations (SST turbulence model) and time dependent simulations (SST-SAS turbulence model) in combination with different calibrated mass transfer models 
each other. The cavitation patterns are represented using the isosurfaces of the vapour volume fraction equal to 0.1 in order to clearly visualize the small (initial) cavitation bubbles predicted at .

Nevertheless, in the case of standard RANS simulations the efficiency was under-predicted, even though the shape of the predicted sigma brake curve compared well with the experimental trend. It is worth noting that the differences in predicted efficiency values were, mainly, due to the over estimation of the draft tube losses related to the steady state approach and turbulence modelling rather than on cavitation modelling [35].

Thus, in order to better resolve the vortex structures in the draft tube and consequently improve the accuracy of the efficiency predictions, additional SAS simulations were performed in combination with the calibrated Zwart mass transfer model.

Following [35] a comparison between the turbulent flow structures predicted by RANS and SAS simulations are presented in Fig. 10.

It is possible to note that for the steady state RANS simulations, only large turbulent structures were obtained.

In the case of SAS simulations, as expected, smaller turbulent structures were resolved leading to more accurate predictions of the draft tube losses.

In Fig. 10 it is also interesting to note the lower level of the viscosity ratio (ratio between the turbulent
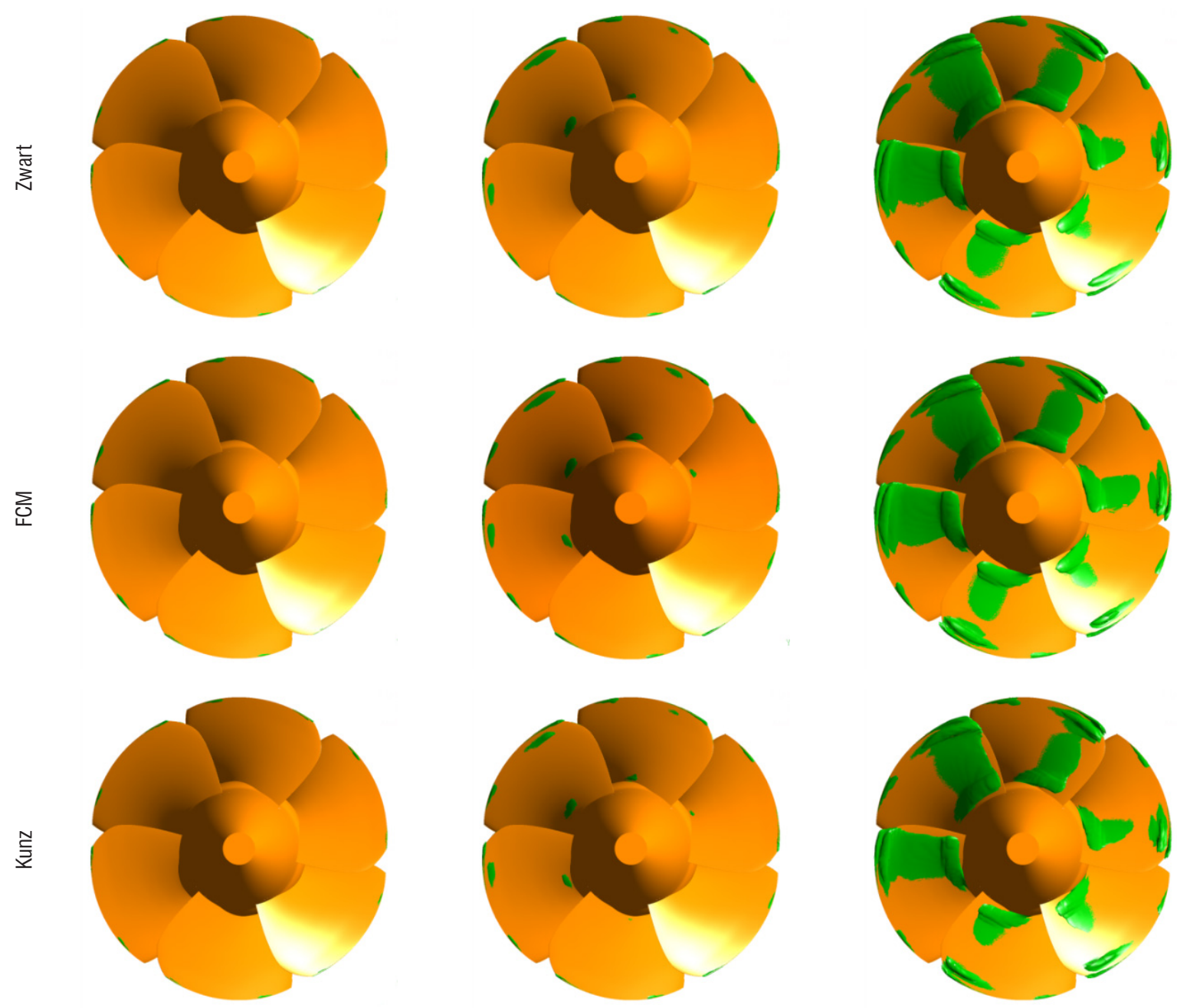

$$
\sigma=0.61
$$

$$
\sigma=0.56
$$

$$
\sigma=0.49
$$

Fig. 8. Kaplan turbine; steady state simulations performed using the SST turbulence model in combination with three different calibrated mass transfer models; cavitation patterns depicted as isosurfaces of the vapour volume fraction equal to 0.1. 


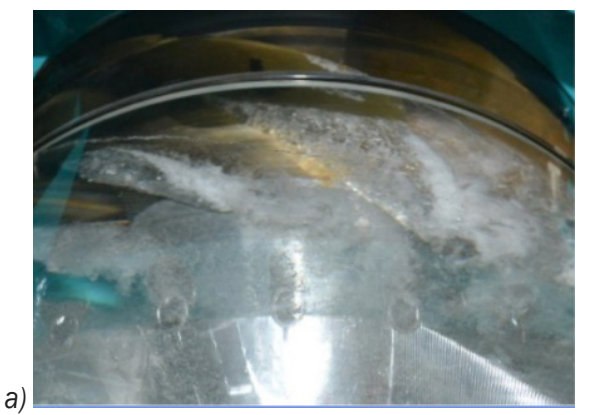

b)

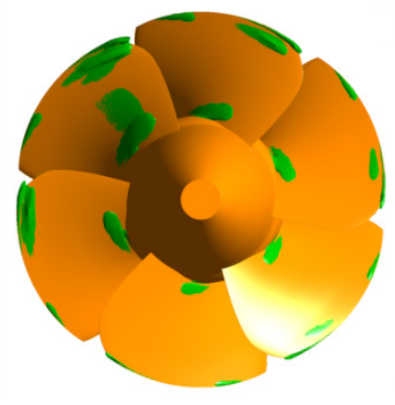

c)

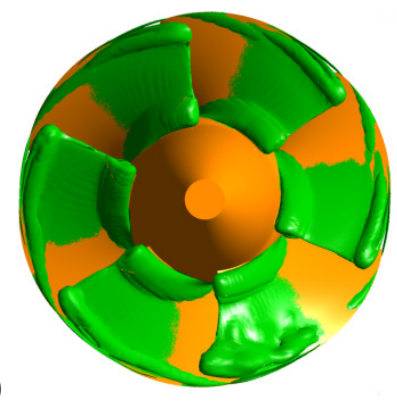

Fig. 9. Cavitation patterns for $\sigma=0.52$; a) experimental recording, b) predicted using Zwart mass transfer model in combination with the SST turbulence model, c) predicted using Zwart mass transfer model in combination with the SST-SAS turbulence model. Numerical cavitation patterns depicted as isosurfaces of vapour volume fraction equal to 0.1 .

a)

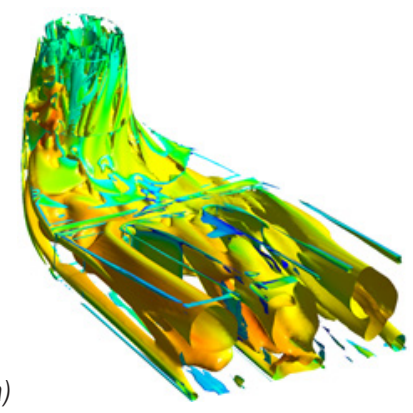

b)

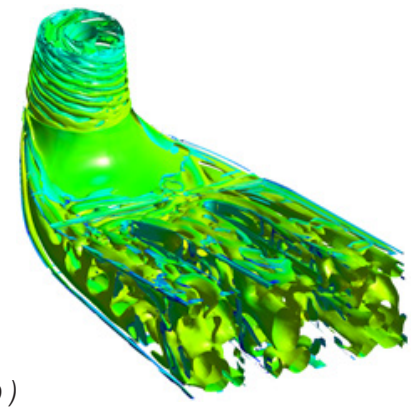

Viscosity Ratio

$1 e+000 \quad 1 e+001 \quad 1 e+002 \quad 1 e+003 \quad 1 e+004$

Fig. 10. Turbulent structures represented using the isosurfaces of velocity invariant equal to 0.1 , coloured by viscosity ratio; a) simulation performed with the SST turbulence model, and b) with the SST-SAS model

and dynamic viscosity) associated with the SAS simulation.

Regarding the efficiency, from Fig. 7, it is possible to note that with the SAS simulations the predicted values compared well with the experimental data even though a premature break down of the turbine performances was predicted.

In Fig. 9 the cavitation patterns obtained with RANS and SAS simulations are qualitatively compared with the available experimental recording for $\sigma=0.52$. It is possible to note that in the case of the steady state RANS simulation the extension of the cavitation phenomenon was under-predicted. This is related to the over prediction of the draft tube losses which lead to an inaccurate pressure distribution in the turbine. Actually, in the case of the steady state RANS simulations for a given cavitation number the pressure in the runner region was higher compared to the experimental one and consequently the predicted cavitation phenomenon was less severe.

Finally, it is worth clarifying that steadystate simulations did not predict the same extent of cavitation on all blades due to the frozen rotor conditions, imposed at Guide Vanes-Runner and Runner-Draft tube interfaces, which somehow preserved differences in circumferential direction.
With transient simulations, as expected, the same amount of vapour structures was obtained on all runner blades.

\section{CONCLUSIONS}

In this study a homogeneous multiphase model, used in combination with three previously calibrated mass transfer models, was evaluated for the numerical prediction of the cavitating flow around a marine propeller and Kaplan turbine runner.

The simulations were carried out considering two different levels of turbulence modelling: the industrial workhorse steady state RANS approach and the more advanced unsteady SAS approach. In both the cases the governing equations were solved using ANSYSCFX 15 commercial CFD solver.

The numerical results were compared with the available experimental data.

For the propeller, the thrust obtained with the RANS simulations, performed along with the three different mass transfer models, compared well with the available experimental data even though the experimental cavitation patterns were not perfectly matched. Except for a particular flow condition the cavitation patterns associated with the different mass 
transfer models were very similar to each other. From an additional investigation performed using the SAS approach a better resolution of the cavitating tipvortex flow was obtained.

Also for the Kaplan turbine the three different mass transfer models predicted similar shapes of the cavitation patterns in the case of the RANS simulations. Nevertheless, using the RANS approach, mainly due to the overestimation of the draft tube losses, the turbine efficiency was not properly predicted. Better prediction of the draft tube losses, as well as of the efficiency, was obtained using the SAS approach even though in this case a premature break down of the performance was obtained.

From the overall results it seems that the calibrated mass transfer models in question can be successfully applied to the numerical predictions of the cavitating flow around a marine propeller and Kaplan turbine. It seems that for the prediction of the machine's performance they can guarantee similar levels of accuracy even though differences in the predicted cavitation patterns can be observed.

Finally, from this study it emerges that for improving the accuracy of numerical predictions, SAS simulations could represent a good compromise between standard RANS simulations and the computationally more demanding and more accurate large eddy simulations (LES).

\section{ACKNOWLEDGEMENTS}

The research leading to these results received funding from the People Programme (Marie Curie Actions) of the European Union's Seventh Framework Programme FP7/2007-2013/ under REA grant agreement $n^{\circ} 612279$. From the Slovenian Research Agency ARRS - Contract No. 1000-15-0263.

\section{NOMENCLATURE}

$C_{e} \quad$ empirical coefficient (FCM model),

$C_{c} \quad$ empirical coefficient (FCM model),

$C_{\text {dest }} \quad$ empirical coefficient (Kunz model),

$C_{\text {prod }}$ empirical coefficient (Kunz model),

$F_{e} \quad$ empirical coefficient (Zwart model),

$F_{c} \quad$ empirical coefficient (Zwart model),

$D$ propeller, turbine runner diameter, [m]

$E \quad$ hydraulic energy, $\left[\mathrm{m}^{2} / \mathrm{s}^{2}\right]$

$H$ turbine head, [m]

$J$ propeller advance coefficient,

$K_{T} \quad$ propeller thrust coefficient,

$L \quad$ characteristic length scale, [m]

$M \quad$ turbine torque, $[\mathrm{Nm}]$
$P \quad$ pressure, $[\mathrm{Pa}]$

$P_{v} \quad$ saturation vapour pressure, $[\mathrm{Pa}]$

$P_{\text {outlet }} \quad$ pressure on outlet boundary, $[\mathrm{Pa}]$

$Q \quad$ flow rate, $\left[\mathrm{m}^{3} / \mathrm{s}\right]$

$R_{n u c} \quad$ radius of a nucleation site, [m]

$S_{M} \quad$ source term,

$\mathrm{T}$ propeller thrust, [N]

U velocity, $[\mathrm{m} / \mathrm{s}]$

$\mathrm{U}_{\infty} \quad$ free stream velocity, $[\mathrm{m} / \mathrm{s}]$

$V_{A} \quad$ propeller advance velocity, $[\mathrm{m} / \mathrm{s}]$

$c_{0.7} \quad$ chord length (at $\left.0.7 /(\mathrm{D} / 2)\right),[\mathrm{m}]$

$f_{v} \quad$ vapour mass fraction,

$g \quad$ gravity acceleration, $\left[\mathrm{m} / \mathrm{s}^{2}\right]$

$k \quad$ turbulence kinetic energy, $\left[\mathrm{m}^{2} / \mathrm{s}^{2}\right]$

$\dot{m} \quad$ mass transfer rate, $\left[\mathrm{kg} /\left(\mathrm{m}^{3} \mathrm{~s}\right)\right]$

$\dot{m}^{+} \quad$ mass transfer rate, vapour to liquid, $\left[\mathrm{kg} /\left(\mathrm{m}^{3} \mathrm{~s}\right)\right]$

$\dot{m}^{-} \quad$ mass transfer rate, liquid to vapour, $\left[\mathrm{kg} /\left(\mathrm{m}^{3} \mathrm{~s}\right)\right]$

$n \quad$ rotational speed, [rps]

$r_{\text {nuс }} \quad$ nucleation site volume fraction,

$t_{\infty} \quad$ mean flow time scale, [s]

$\rho \quad$ mixture density, $\left[\mathrm{kg} / \mathrm{m}^{3}\right]$

$\rho_{l}, \rho_{v} \quad$ liquid density, vapour density, $\left[\mathrm{kg} / \mathrm{m}^{3}\right]$

$\eta \quad$ turbine efficiency,

$\varphi \quad$ turbine flow coefficients,

$\varphi_{B E P}$ turbine flow coefficients at local best efficiency point,

$\alpha \quad$ vapour volume fraction,

$\gamma \quad$ liquid volume fraction,

$\kappa \quad$ surface tension, $[\mathrm{N} / \mathrm{m}]$

$\mu \quad$ mixture dynamic viscosity, [Pa s]

$\mu_{l} \quad$ liquid dynamic viscosity, [Pa s]

$\mu_{v} \quad$ vapour dynamic viscosity, [Pa s]

$\sigma \quad$ turbine cavitation number,

$\sigma_{n} \quad$ propeller cavitation number,

$\tau$ stress tensor, [N/m²]

$\omega \quad$ turbulence frequency, [1/s]

$\psi \quad$ turbine energy coefficient

$\psi_{B E P}$ turbine energy coefficient at local best efficiency point

NPSE net positive suction energy, $\left[\mathrm{m}^{2} / \mathrm{s}^{2}\right]$

\section{REFERENCES}

[1] Keck, H., Sick, M. (2008). Thirty years of numerical flow simulation in hydraulic turbomachines. Acta Mechanica, vol. 201, no. 1-4, p. 211-229, D0I:10.1007/s00707-008-0060-4.

[2] Stern, F., Wang, Z., Yang, J., Sadat-Hosseini, H., Mousaviraad, M., Bhushan, S., Diez, M., Yoon, S.-H., Wu, P.-C., Yeon, S.M., Dogan, T., Kim, D.-H., Volpi, S., Conger, M., Michael, T., Xing, T., Thodal, R.S., Grenestedt, J.L. (2015). Recent progress in CFD for naval architecture and ocean engineering. Journal of Hydrodynamics, Ser. B, vol. 27, no. 1, p. 1-23, D0l:10.1016/ S1001-6058(15)60452-8. 
[3] Luo, X., Ji, B., Tsujimoto, Y. (2016). A review of cavitation in hydraulic machinery. Journal of Hydrodynamics, Ser. B, vol. 28, no. 3, p. 335-358, D0I:10.1016/S1001-6058(16)60638-8.

[4] Štefan, D., Rudolf, P., Muntean, S., Susan-Resiga, R. (2017). Proper orthogonal decomposition of self-induced instabilities in decelerated swirling flows and their mitigation through axial water injection. Journal of Fluids Engineering, vol. 139, no. 8, p. 081101, D0l:10.1115/1.4036244.

[5] Ji, B., Luo, X., Wu, Y. (2014). Unsteady cavitation characteristics and alleviation of pressure fluctuations around marine propellers with different skew angles. Journal of Mechanical Science and Technology, vol. 28, no. 4, p. 1339-1348, DOI:10.1007/s12206-013-1166-8.

[6] Morgut, M., Nobile, E. (2012). Numerical predictions of cavitating flow around model scale propellers by CFD and advanced model calibration. International Journal of Rotating Machinery, vol. 2012, article id 618180, DOl:10.1155/2012/618180.

[7] Young, F.R. (1999). Cavitation. Imperial College Press, London, DOI:10.1142/p172.

[8] Rus, T., Dular, M., Širok, B., Hočevar, M., Kern, I. (2007). An investigation of the relationship between acoustic emission, vibration, noise, and cavitation structures on a Kaplan turbine. Journal of Fluids Engineering, vol. 129, no. 9, p. 1112-1122, DOI:10.1115/1.2754313.

[9] Jian, W., Petkovšek, M., Houlin, L., Širok, B., Dular, M. (2015). Combined numerical and experimental investigation of the cavitation erosion process. Journal of Fluids Engineering, vol. 137, no. 5, p. 051302, Dol:10.1115/1.4029533.

[10] Dular, M., Petkovšek, M. (2015). On the mechanisms of cavitation erosion - Coupling high speed videos to damage patterns. Experimental Thermal and Fluid Science, vol. 68, p. 359-370, D0I:10.1016/j.expthermflusci.2015.06.001.

[11] Wittekind, D., Schuster, M. (2016). Propeller cavitation noise and background noise in the sea. Ocean Engineering, vol. 120, p. 116-121, D0I:10.1016/j.oceaneng.2015.12.060.

[12] Carlton, J.S. (2012). Marine Propellers and Propulsion (3rd Ed.). Butterworth-Heinemann, Oxford.

[13] Koop, A. H. (2008). Numerical Simulation of Unsteady TreeDimensional Sheet Cavitation. PhD thesis. University of Twente, Twente.

[14] Tran T.D., Nennemann, B., Vu, T.C., Guibald F. (2015). Investigation of cavitation models for steady and unsteady cavitating flow simulation. International Journal of Fluid Machinery and Systems, vol. 8, no. 4, p. 240-253, DOI:10.5293/IJFMS.2015.8.4.240.

[15] Senocak, I., Shyy, W. (2004). Interfacial dynamics-based modeling of turbulent cavitating flows, Part-1: Model development and steady-state computations. International Journal of Numerical Methods in Fluids, vol. 44, no. 9, p. 975995, D0I:10.1002/fld.692.

[16] Asnaghi, A., Feymark, A., Bensow, R.E. (2017). Improvement of cavitation mass transfer modeling based on local flow properties. International Journal of Multiphase Flow, vol. 93, p. 142-157, D0l:10.1016/j.ijmultiphaseflow.2017.04.005.

[17] Zwart, P., Gerber, A.G., Belamri, T. (2004). A two-phase model for predicting cavitation dynamics. ICMF International Conference on Multiphase Flow, Yokohama.
[18] Singhal, A.K., Athavale, M.M., Li,H., Jiang, Y. (2002). Mathematical basis and validation of the full cavitation model. Journal of Fluids Engineering, vol. 124, no. 3, p. 617-624, DOI:10.1115/1.1486223.

[19] Kunz, R.F. , Boger, D.A., Stinebring, D.R., Chyczewski, T.S., Lindau, J.W., Gibeling, H. J., Venkateswaran, S., Govindan, T.R. (2000). A preconditioned Navier-Stokes method for two-phase flows with application to cavitation prediction. Computers and Fluids, vol. 29, no. 8, p. 849-875, D0l:10.1016/S00457930(99)00039-0.

[20] Morgut, M., Nobile, E. and Biluš, I. (2011). Comparison of mass transfer models for the numerical prediction of sheet cavitation around a hydrofoil. International Journal of Multiphase Flow, volume 37, no. 6, p. 620-626, D0l:10.1016/j. ijmultiphaseflow.2011.03.005.

[21] SVA Schiffbau-Versuchsanstalt Potstdam (2011). PPTC smp'11 Workshop, from http://www.sva-potsdam.de/en/ pptc-smp11-workshop, accessed on 2017-03-02.

[22] Menter, F.R. (1994). Two-equation eddy-viscosity turbulence models for engineering applications. AIAA Journal, vol. 32, no 8, p. 1598-1605, DOl:10.2514/3.12149.

[23] Egorov, Y., Menter. F. (2007). Development and Application of SST-SAS Turbulence Model in the DESIDER Project. Proceedings of the $2^{\text {nd }}$ Symposium on Hybrid RANS-LES Methods, Corfu.

[24] Schneider, G., Raw, M. (1987). Control volume finiteelement method for heat transfer and fluid flow using collocated variables - 1. Computational procedure. Numerical Heat Transfer, vol. 11, no. 4, p. 363-390, DOI:10.1080/10407788708913560.

[25] Schneider, G., Raw, M. (1987). Control volume finiteelement method for heat transfer and fluid flow using collocated variables - 2. Application and validation. Numerical Heat Transfer, vol. 11, no. 4, p. 391-400, DOI:10.1080/10407788708913561.

[26] ANSYS (2013). CFX Solver Theory Guide, Release 15.0, Canonsburg.

[27] Huuva, T. (2008). Large Eddy Simulation of Cavitating and Non-Cavitating Flow. Ph.D. Thesis, Chalmers University of Gothenburg, Gothenburg.

[28] Versteeg, H.K., Malalasekera, W. (2007). An Introduction to Computational Fluid Dynamics: The Finite Volume Method, (2nd Ed.). Pearson Education Limited, London.

[29] Morgut, M., Nobile, E. (2012). Influence of grid type and turbulence model on the numerical prediction of flow around marine propellers working in uniform inflow. Ocean Engineering, vol. 42, p. 26-34, Dol:10.1016/j. oceaneng.2012.01.012.

[30] Morgut, M., and Nobile, E. (2011). Numerical predictions of the cavitating and non-cavitating flow around the model scale propeller PPTC. Proceedings of Workshop on Cavitation and Propeller Performance, Second International Symposium on Marine Propulsors, smp'11, Hamburg, Germany.

[31] IEC 60193 (1999). Hydraulic Turbines, Storage Pumps and Pump-Turbine - Model Acceptance Tests. International Electrotechnical Commission, Geneva.

[32] Smirnov, P.E., Menter, F.R. (2009). Sensitization of the SST turbulence model to rotation and curvature by applying the 
Spalart-Shur correction term. Journal of Turbomachinery, vol. 131, no. 4, p. 041010, D0l:10.1115/1.3070573.

[33] Kato, M., Launder, B.E. (1993). The modelling of turbulent flow around stationary and vibrating square cylinders. Proceedings of the Ninth Symposium on Turbulent Shear Flows, p. 10.4.110.4.6.

[34] Jošt, D., Škerlavaj, A., Lipej, A. (2014). Improvement of efficiency prediction for a Kaplan turbine with advanced turbulence models. Strojniški vestnik - Journal of Mechanical Engineering, vol. 60, no. 2, p. 124-134, Dol:10.5545/svjme.2013.1222.

[35] Jošt, D., Morgut, M., Škerlavaj, A., Nobile, E. (2015). Cavitation prediction in a Kaplan turbine using standard and optimized model parameters. Proceedings of $6^{\text {th }}$ IAHR International Meeting of the Workgroup on Cavitation and Dynamic Problems in Hydraulic Machinery and Systems, Ljubljana. 